\title{
Nicotine Withdrawal and Psychiatric Symptoms in Cigarette Smokers with Schizophrenia
}

\author{
Gregory W. Dalack, M.D., Lisa Becks, M.S.W., Elizabeth Hill, Ph.D., Ovide F. Pomerleau, Ph.D., \\ and James H. Meador-Woodruff, M.D.
}

The prevalence of smoking is markedly elevated in schizophrenia. Low smoking cessation rates and reports that some smokers with schizophrenia experience an acute increase in symptoms during attempts to quit smoking, suggest a self-medication model. Alternatively, smoking may modulate medication side effects. The effects of treated and untreated smoking abstinence on psychotic symptoms and medication side effects were examined in this study. Nineteen outpatients with schizophrenia or schizoaffective disorder participated in a randomized, double-blind, balanced crossover study: 1 day of ad libitum smoking followed by 3 days of acute smoking abstinence while wearing $22 \mathrm{mg} /$ day active or placebo transdermal nicotine patches, with a return to 3 days of smoking between patch conditions. Daily symptom and side-effect ratings, nicotine and cotinine blood levels were collected. Twelve subjects completed the study. Neither positive symptoms nor mood symptoms changed. An increase in negative symptoms during the first abstinent day occurred in both placebo and active patch conditions, but was not sustained over subsequent abstinent days. Despite physiological signs of withdrawal, completers did not endorse increased nicotine withdrawal symptoms. Dropouts reported higher withdrawal symptoms, but also had no increase in psychiatric symptoms in either phase of the study. Of note, dyskinesias decreased during abstinence and placebo patch treatment, but increased during abstinence and the active patch conditions. Acute exacerbation of psychiatric symptoms is an unlikely explanation for any difficulty smokers with schizophrenia have in early abstinence.

[Neuropsychopharmacology 21:195-202, 1999] (C) 1999 American College of Neuropsychopharmacology. Published by Elsevier Science Inc.
KEY WORDS: Cigarette smoking; Nicotine; Schizophrenia; Nicotine Withdrawal

It is well known that the prevalence of smoking among those with schizophrenia is two to three times higher than that in the general population and considerably higher than that among those with other psychiatric illnesses (Hughes et al. 1986; de Leon et al. 1995). Cross-

From the Ann Arbor VA Medical Center (GWD) and Department of Psychiatry (GWD, LB, EH, OFP, JHM-W), University of Michigan, Ann Arbor, Michigan.

Address correspondence to: G. W. Dalack, M.D., 2215 Fuller Road

116C, Ann Arbor, MI 48105.

Received February 24, 1998; accepted August 10, 1998. sectional studies have suggested that smoking may be a marker of more severe schizophrenic illness and may affect treatment by modulating side effects of antipsychotic medication (Sandyk and Kay 1991; Goff et al. 1992). For example, neuroleptic-induced parkinsonism (NIP) is often found to be less prevalent among schizophrenics who smoke (Decina et al. 1990), whereas, the rate of tardive dyskinesia had been variably reported to be higher or lower (Yassa et al. 1987; Menza et al. 1991). The suggested effects of smoking on NIP may be accounted for by the faster metabolic clearance of neuroleptics attributable to hepatic microsomal enzyme upregulation caused by the products of tobacco combustion (Benowitz 1988), although some studies sug- 
gest that higher doses of medication are required for smokers versus nonsmokers treated with neuroleptics (Ereshefsky et al. 1985; Jann et al. 1986).

A number of explanations for the extensive cooccurrence of smoking with schizophrenia have been forwarded (Freedman et al. 1997; Glassman 1993). Some have focused on the ameliorating effects of nicotine on attentional abnormalities found in schizophrenia (Freedman et al. 1997; Adler et al. 1993), whereas, others have posited that the effects of nicotine on reward reinforcement and negative symptoms of schizophrenia are important (Glassman 1993; Nisell et al. 1995). Systematic studies of the effects of nicotine withdrawal have not been reported.

The available cessation data suggest that smoking cessation rates among smokers with schizophrenia are quite low (Covey et al. 1994; Addington et al. 1997; Ziedonis and George 1997). In part, this may relate to lower motivation to quit (Ziedonis and George 1997). We have reported cases that suggested that attempts to cut down or quit smoking led to an exacerbation of psychiatric symptoms and a return to smoking (Dalack and Meador-Woodruff 1996).

The very high prevalence of smoking among those with schizophrenia, coupled with our clinical impression that smoking abstinence may to lead to an exacerbation of psychiatric symptoms, led us to consider whether smoking in schizophrenia might be an attempt to self-medicate symptoms of the illness or side effects of treatment. Accordingly, we used an experimental paradigm that most closely paralleled our clinical observations. We conducted a randomized, double-blind, placebo-controlled crossover study to examine the effect of acute nicotine withdrawal on psychiatric symptoms and medication side effects in smokers with schizophrenia. Our goal was to examine the following hypotheses.

1. Acute nicotine withdrawal will exacerbate psychiatric symptoms in heavy smokers with schizophrenia.

2. Treatment of acute withdrawal by transdermal nicotine patch will mitigate both the increase in withdrawal symptoms and psychiatric symptoms.

3. Neuroleptic-induced parkinsonism will worsen during acute nicotine withdrawal; tardive dyskinesia will not change.

4. Treatment of acute nicotine withdrawal by transdermal nicotine patch will limit the exacerbation of neuroleptic-induced Parkinsonism.

\section{SUBJECTS}

Subjects with schizophrenia or schizoaffective disorder were recruited for participation in this study through referral from the treating psychiatrist or bulletin board advertising in the Mental Health Clinics at the Ann Arbor $(n=13)$ and Battle Creek $(n=6)$ VA Medical Centers (VAMCs). Subjects were required to be heavy smokers ( $\geqslant 20$ cigarettes per day) and stable on an antipsychotic regimen for at least the previous 3 months. The Structured Clinical Interview for DSM-III-R (Spitzer et al. 1989) was used to confirm the diagnosis of schizophrenia or schizoaffective disorder and absence of current non-nicotine substance use disorders. Urine toxicology screens were obtained on the first day of each phase of the study to confirm absence of current nonnicotine substance use. None of the subjects had a history of serious medical illness. All had a normal physical examination and laboratory tests in the past year and a normal ECG in the last 6 months. The Institutional Review Boards of the Ann Arbor and Battle Creek VAMC and the University of Michigan Medical Center approved this study. All subjects provided written informed consent and were paid for completing each phase of the study ( $\$ 25$ for completing the first phase, $\$ 75$ for completing the second phase).

Nineteen subjects entered the study. They were middle-aged (40 \pm 5 years) male veterans, predominantly white $(68 \%)$, with a diagnosis of schizophrenia $(74 \%)$ or schizoaffective disorder (26\%). They had been ill for $17.8 \pm 7.3$ years. Ninety-five percent had a high school education or more. Most were single, and all received disability entitlements for their condition. Nearly $75 \%$ were on typical antipsychotics (mean daily dose in chlorpromazine equivalents [Davis 1976]: $1221 \pm 1092$ $\mathrm{mg}$ ); the rest were taking clozapine (mean dose: $435=$ $176 \mathrm{mg}$ ). On average, they were heavy smokers (cigarettes per day: $29.4 \pm 18.7)$ who reported multiple quit attempts $(3.8 \pm 1.6)$. Their level of nicotine addiction was moderate to severe (Fagerstrom Test for Nicotine Dependence [Heatherton et al. 1991] score: $6.2 \pm 2.4$ ). They had a baseline (4-5 PM) nicotine level of $15.2 \pm 8.7$ $\mathrm{ng} / \mathrm{ml}$ and cotinine level of $389 \pm 116 \mathrm{ng} / \mathrm{ml}$.

\section{METHODS}

\section{Nicotine Patches/Nicotine Plasma Levels}

Active nicotine transdermal patches (22 $\mathrm{mg}$ /day) were purchased from Lederle Inc. (Prostep ${ }^{\circledR}$, Pearl River, New York). Placebo patches, prepared by the Investigational Pharmacy Service at the University of Michigan Hospital, were fashioned by removing the nicotineinfused agarose plug from the center of the patch and replacing the foil barrier that makes contact with the skin. Nicotine and cotinine plasma levels were determined by an high-performance liquid chromatography (HPLC) assay (Hariharan et al. 1988). Plasma levels confirmed that placebo patches delivered negligible quantities of nicotine.

\section{Behavioral Ratings}

Ratings of psychiatric symptoms and medication side effects were assessed using: Brief Psychiatric Rating 
Scale (BPRS, Overall and Gorham 1962), Schedule for the Assessment of Negative Symptoms (SANS, Andreasen 1982), Hamilton Scale for Depression (HAM-D, Hamilton 1960), Simpson-Angus Scale (SAS, Simpson and Angus 1970), and Abnormal Involuntary Movement Scale (AIMS, Psychopharmacology Research Branch, National Institute of Mental Health 1976; Munetz and Benjamin 1988). The majority of the ratings were carried out by one of us (LB). Inter-relater reliability for scale data was calculated using intraclass correlation coefficient (ICC) and indicated good levels of agreement (e.g., BPRS ICC $=0.71$; SANS ICC $=0.75$ ). Nicotine withdrawal symptoms were assessed using a version of the Hughes-Hatsukami Scale (Hughes and Hatsukami 1986). In addition to total BPRS and SANS scores, subscale scores for each were also calculated (Andreasen 1982; Krystal et al. 1994; Hedlund and Vieweg 1980). For the BPRS, the subscales were: (1) positive symptoms (conceptual disorganization, hallucinatory behavior, suspiciousness, unusual thought content); (2) negative symptoms (blunted affect, emotional withdrawal, motor retardation); (3) activation (tension, mannerisms, excitement); and (4) hostility (hostility, suspiciousness, uncoopertiveness, grandiosity). For the SANS, six subscales of individual domains were determined by calculating the sum of items (not including the global rating) for each domain (affective flattening, alogia, avolition/apathy, anhedonia-asociality, attention), and a global item calculated as the sum of the global ratings from each domain. At baseline, subjects were psychiatrically stable with moderate levels of symptomatology (baseline ratings: BPRS $33.5 \pm 7.2$; SANS $28.2 \pm 13.7$; HAM-D $6.4 \pm 4.2$; AIMS $3.5 \pm 2.7$; SAS $2.3 \pm 1.5)$.

\section{Protocol}

A double-blind, placebo-controlled, counterbalanced crossover design was used. Subjects were admitted to the Clinical Research Center (CRC) at the University of Michigan Hospital for two 4-day stays, with at least 3 days in between. Upon admission to the CRC, subjects smoked ad libitum for 1 day (no blind), followed by acute abstinence (over 3 days) while wearing active or placebo transdermal nicotine patch (double-blind). At the end of the first 4-day stay, subjects returned home to smoke ad libitum, without patches, for at least 3 days. They returned the following week to repeat the procedure ( 1 day of ad libitum smoking, followed by 3 days of abstinence) in the other patch condition. The standardized clinical ratings battery was performed each day in the CRC to assess psychiatric symptomatology, medication side effects, and symptoms of nicotine withdrawal. In addition, vital signs were monitored daily, and nicotine and cotinine blood levels were drawn each afternoon between 4 and 5 PM in the CRC. Smoking was not permitted in the CRC. During the ad libitum smoking days, subjects were escorted by research staff to appropriate smoking areas around the hospital. Subjects surrendered cigarettes and other smoking paraphernalia at the end of the ad libitum smoking day. Research and nursing staff periodically checked on subjects during the day, and research staff accompanied subjects during walks off of the research unit. Similarly, nursing staff periodically checked on subjects during evening and night shifts. Carbon monoxide in expired air was measured in the afternoon on the ad libitum smoking day and the last day of abstinence in each phase. Clinically prescribed pharmacological treatment regimens were continued throughout the study.

\section{Data Analysis}

Daily ratings were analyzed by repeated measures analysis of variance (ANOVA) (subjects/patch order $\times$ patch status $\times$ day), as well as an intent to treat analysis (SAS Software 6.11, SAS Institute Inc., Cary, NC). Withdrawal symptoms (total withdrawal symptom score and craving item alone), psychiatric symptoms (BPRS, SANS, SAS, AIMS, HAM-D total scores; BPRS and SANS subscale scores), vital signs and nicotine and cotinine blood levels were the dependent measures. Nonsignificant effects are not presented $(p>.05)$ unless they were of specific interest. Distributions of dependent measures were checked for normality, and some analyses were performed after log transformation. A further set of analyses was run using dependent measures transformed to relative change from baseline. However, because the pattern of results did not change with transformations, analyses of raw measures are presented. In the analysis of heart rate, three missing datapoints (one in each of three subjects) were imputed using the mean for the group, on the day and patch condition in which the missing value occurred.

Nineteen subjects entered the study. Twelve completed the protocol, and three more participated in some part of both phases of the study. The intent to treat analysis $(n=19)$ and the repeated measures ANOVA results for completers only $(n=12)$ were essentially the same. Hence, the results for the analysis of completers are presented below. Insofar as there was no main effect for patch order, all placebo phase data were compared to all active phase data, regardless of the order of patch type.

\section{RESULTS}

\section{Smoking Abstinence/Withdrawal Symptoms}

As shown in Figure 1, heart rate dropped for subjects during untreated withdrawal. Carbon monoxide in expired air (CO) dropped to nonsmoker levels during nonsmoking periods [mean $\mathrm{CO} \pm \mathrm{SD}(\mathrm{pm})$ for ad libitum smoking day vs. last abstinent day: $30.7 \pm 14.0$ vs. 
$1.2 \pm 1.2]$. Nicotine blood levels decreased during the placebo phase, as would be expected (Figure 1). Because the transdermal patch does not fully replace nicotine obtained by smoking, nicotine levels decreased somewhat and stabilized at a lower level during the active patch condition. A review of the nicotine blood level data suggested that only one subject managed surreptitious smoking in the CRC during the placebo phase of the study. However, this subject's CO level did not increase significantly, and his cotinine level dropped during each successive day of the placebo phase. Hence, the amount smoked would seem to be small. We analyzed the completer data excluding this subject, and the findings were not changed. Temperature and blood pressure measurements were unchanged in both placebo and active patch phases of the study. Despite nicotine abstinence and physiological evidence of withdrawal, mild increases in reported subjective withdrawal symptoms did not reach statistical significance in either condition.

\section{Psychiatric Symptoms}

Daily total BPRS and SANS scores are shown in Figure 2. There was no significant change in total BPRS score. There was a trend increase in the negative symptoms subscale score of the BPRS [main effect of day: $\mathrm{F}(3,30)=$ $2.48, p<.09$ ], which paralleled the SANS score change (see below). In addition, the BPRS activation subscale showed a decrease during the first smoking abstinence day [main effect of day: $\mathrm{F}(3,30)=4.55, p<.02$ ], with a return to baseline over subsequent days. Total SANS demonstrated a significant change in both phases of the study, which was unrelated to patch status. This effect seemed to be carried by an increase in total SANS score on the first abstinent day, with a return to essentially baseline scores during the following 2 abstinent days (see Figure 2). Similarly, the SANS subscale ratings showed parallel, significant changes in affective flattening, avolition/apathy, and global subscales, but no changes in alogia, anhedonia-asociality, or attention subscales. HAM-D scores showed no change with respect to patch status or time.

Six subjects dropped out during or at the end of the first period of the study. Four of these had been on placebo patch, two were on active. Of the four dropouts during the placebo phase, two returned to complete the active patch phase. One subject dropped out in both weeks [day 3 of 4 during first phase (active patch) and day 2 of 4 during the second phase (placebo phase)]. More dropouts occurred during the placebo versus the active phase of the study ( 5 vs. 3 , plus 1 subject entered and dropped out of both phases of the study). Dropouts endorsed a significantly higher level of withdrawal symptoms on the day of dropout, as compared with withdrawal scores of completers on the same day (28.3 \pm 11.9 vs. $13.3 \pm 9.1 ; \mathrm{df}=13 ; p<.03$ ). Despite this, dropouts had no increase in psychiatric symptoms during placebo or patch phases of the study (e.g., placebo-phase BPRS day 1 vs. day 3: $34.0 \pm 9.5$ vs. $35.0 \pm 11.5 ; p=N S$ ). None of the dropouts endorsed withdrawal symptoms as the reason for ending participation in the study.
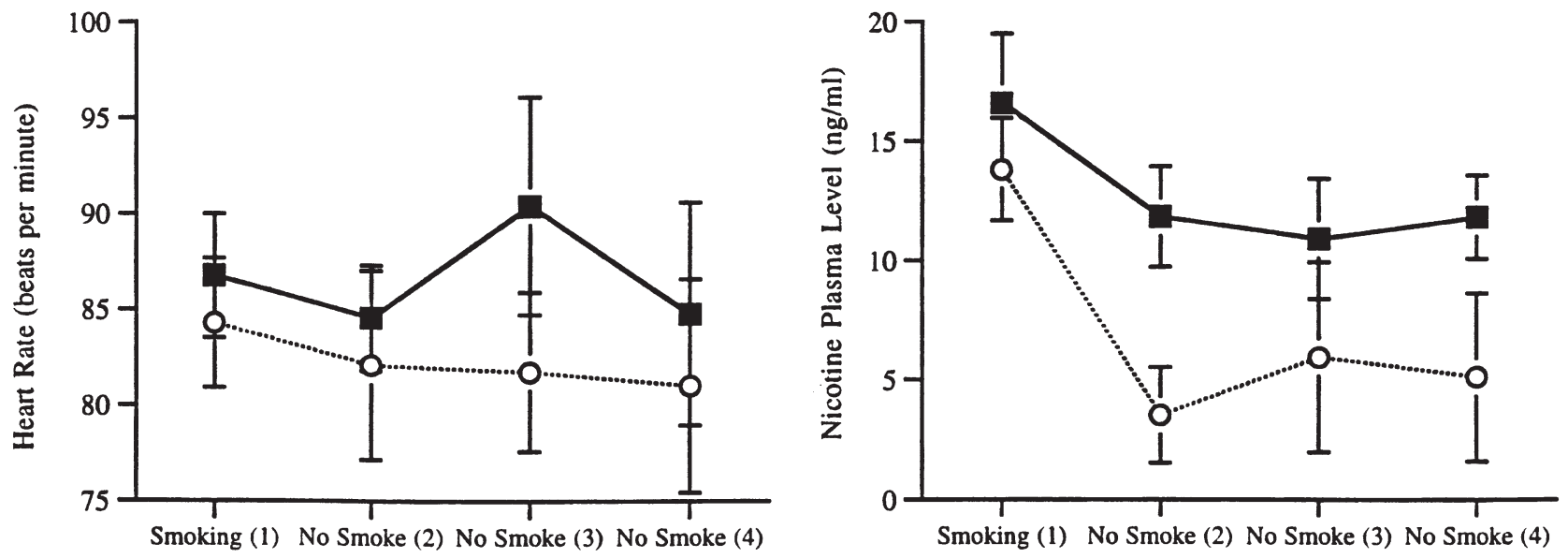

\section{Smoking Status}

Figure 1. Left, the effects of nicotine withdrawal with patch treatment on resting heart rate in 12 completers. Morning heart rates (mean $\pm \mathrm{SE}$ ) are presented for placebo patch (open circles) and active patch (closed squares). Day 1 is ad libitum smoking day (no patch). Repeated measures ANOVA main effect of patch: $\mathrm{F}(1,10)=9.89, p<.02$ ). Right, the effects of nicotine withdrawal with patch treatment on afternoon nicotine plasma levels in 12 completers. Plasma levels (mean \pm SE) are presented for placebo patch (open circles) and active patch (closed squares). Day 1 is ad libitum smoking day (no patch). Repeated measures ANOVA main effects of patch $[\mathrm{F}(1,10)=15.16, p<.05)$ and day $[\mathrm{F}(3,30)=6.72, p<.005]$. 

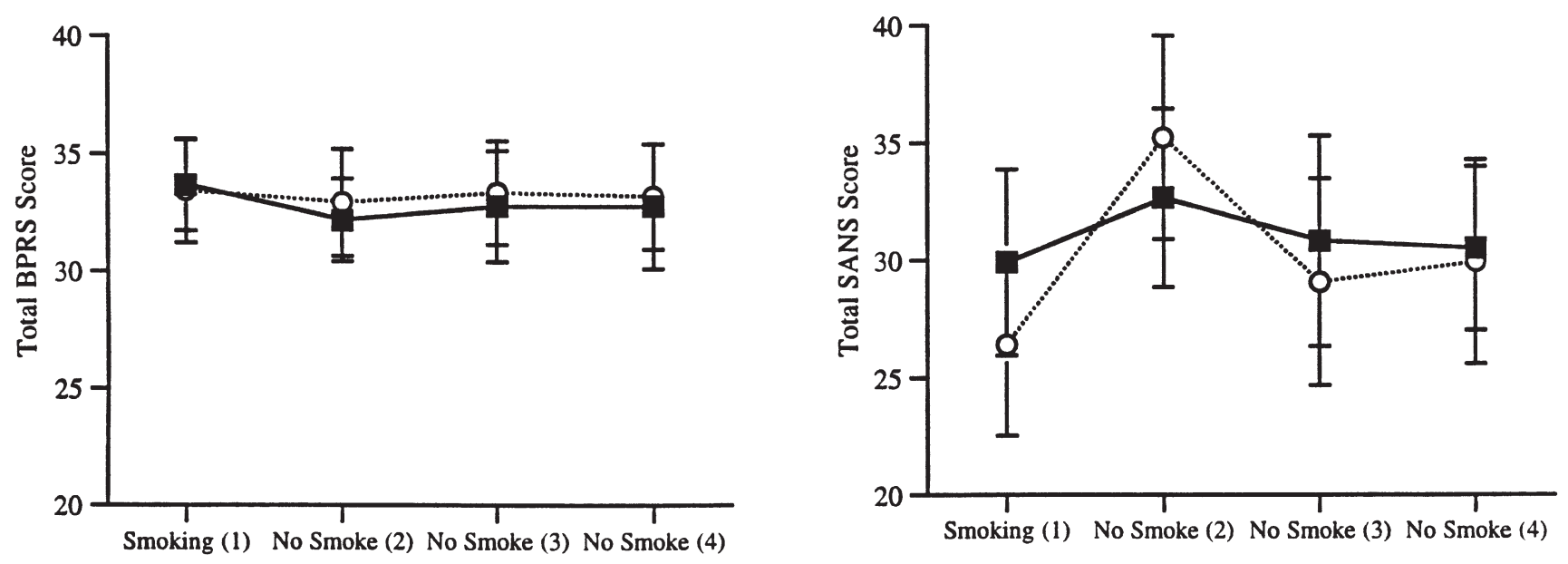

\section{Smoking Status}

Figure 2. Left, the effects of nicotine withdrawal with patch treatment on BPRS total score in 12 completers. Total scores (mean \pm SE) are presented for placebo patch (open circles) and active patch (closed squares). Day 1 is ad libitum smoking day (no patch). Repeated measures ANOVA had no main or interaction effects. Right, the effects of nicotine with patch treatment on SANS total scores in 12 completers. Total scores (mean \pm SE) are presented for placebo patch (open circles) and active patch (closed squares). Day 1 is ad libitum smoking day (no patch). Repeated measures ANOVA main effect of day $[\mathrm{F}(3,30)=4.09 ; p<.02]$.

\section{Medication Side Effects}

Measures of neuroleptic-induced parkinsonism did not change during smoking abstinence with or without nicotine replacement. We did find that abnormal involuntary movements were significantly affected during treated and untreated smoking abstinence (Figure 3). During the placebo patch phase of abstinence, AIMS scores decreased by as much as $35 \%$. During active patch phase, AIMS scores increased by $17 \%$.

\section{DISCUSSION}

Contrary to our expectation, we did not find changes in positive or negative symptoms consistent with a nicotine withdrawal-induced exacerbation during 3 days of treated or untreated smoking abstinence. A "reverse power calculation suggests that our $n$ of 12 provides sufficient power to detect a change of approximately 6 points in total BPRS and 8 points in total SANS scores. Although this level of detection would be sensitive to a modest but still clinically significant symptom change, changes of smaller magnitude cannot be excluded based on our results.

The increase in SANS score as a function of day of study, but not patch status, is of interest. If this increase in SANS score were a function of nicotine withdrawal, we would have expected the increase to persist, and possibly worsen with each day of withdrawal. In fact, SANS scores peaked during the first withdrawal day, and then decreased back toward baseline. Anecdotally, many subjects declined opportunities during the first withdrawal day to leave the CRC with an escort and walk around the hospital, concerned that they would see others smoking and feel the urge to smoke. Hence, we suspect that the tendency to stay in the room, and

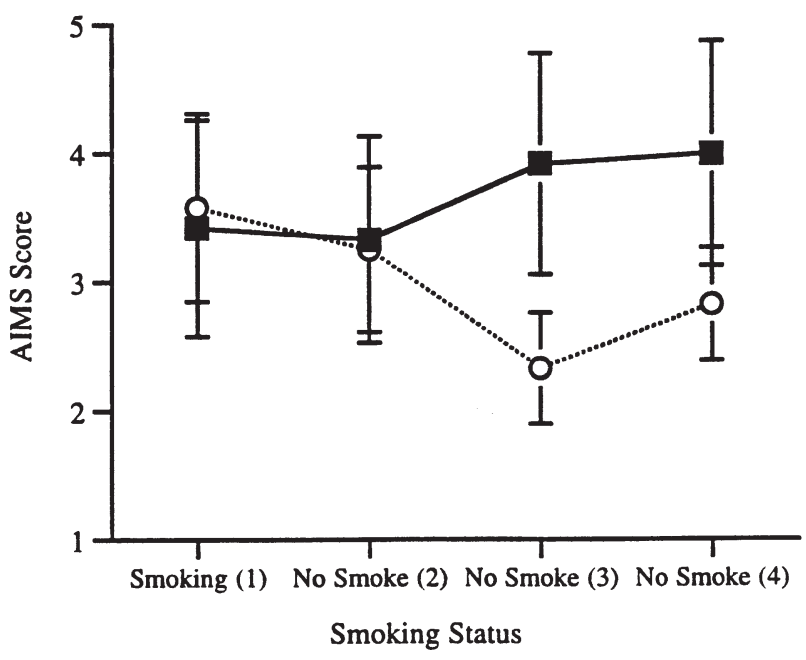

Figure 3. The effects of nicotine withdrawal with patch treatment on AIMS scores in 12 completers. Total scores (sum items 1-7) (mean \pm SE) are presented for placebo patch (open circles) and active patch (closed squares). Day 1 is ad libitum smoking day (no patch). Repeated measures ANOVA interaction effect (patch $\times$ day) $[\mathrm{F}(3,30)=3.84 ; p<.02]$. 
often in bed during the first withdrawal day, led to this SANS increase. The parallel increases in affective blunting and avolition/apathy, but not in alogia, anhedonia, or attention, suggest that the transient increase in negative symptoms was related to behavioral inactivity. The decrease in BPRS activation subscale score also supports this. That the SANS increase occurred with or without nicotine replacement suggests an effect of the act of not smoking more than nicotine withdrawal.

Despite the fact that completers during the placebo phase were without nicotine and demonstrated physiological evidence of withdrawal based on decreased heart rate, they did no report an increase in withdrawal symptoms. In addition, subjects who dropped out endorsed increased withdrawal scores on the day of dropout, but had no increase in psychiatric symptoms and denied that nicotine withdrawal symptoms led to their decision to leave the study. These observations suggest an alteration in the perception of nicotine withdrawal symptoms that may be related to the structured and supportive environment of the CRC, and/or to the distortions of internal apperception that may occur in schizophrenia. The absence of reported withdrawal symptoms among smokers abstaining in hospital settings has been reported for nonpsychiatric smokers (Hughes et al. 1994). It remains unclear, however, whether diagnosis, study environment, or some combination of the two affects this observation.

These findings must be seen in context, and several caveats must be given. Our small sample size permits us to find relatively robust changes in symptoms, certainly of the order of magnitude reported in our case studies, suggesting an exacerbation of psychotic symptoms during nicotine withdrawal (Dalack and MeadorWoodruff 1996). Based on these results, such symptom exacerbation is at least uncommon and is unlikely to explain the difficulty most smokers with schizophrenia have in abstaining.

It has been suggested in nonpsychiatrically ill smokers, that placebo patch treatment may have an effect on response to smoking treatments, which is intermediate between a "no-patch" treatment and active patch (Pickworth et al. 1994). Hence, it is possible that a "no-patch" phase might have increased the likelihood of finding significant changes in symptoms during smoking abstinence.

It is also possible that a longer period of abstinence would result in an exacerbation of symptoms. We picked 3 days of abstinence because of: (1) evidence that nicotine withdrawal peaks over 24 to 48 hours of abstinence (Benowitz 1988; Hatsukami et al. 1984); (2) our anecdotal experience of acute exacerbation of symptoms when patient tried to stop smoking on their own; and (3) our clinical experience that patients are very unlikely to remain abstinent for longer periods of time.
We conclude that exacerbation of symptoms is not an acute abstinence phenomenon.

Finally, it should also be remembered that this study was conducted in stable outpatients. Acutely ill inpatients may respond differently to smoking abstinence and treated or untreated nicotine withdrawal, and generalizations of our findings to an acutely ill inpatient population probably should not be made.

We were unable to find an acute effect of nicotine abstinence on parkinson-like side effects. It may be that any amelioration of such side effects by nicotine persists, even after withdrawal of nicotine itself. There is precedent for such an effect with nicotine augmentation of haloperidol treatment for Tourette's Syndrome (Sanberg et al. 1997).

The threshold for nicotine effects on extrapyramidal syndromes may also be different for various movement disorders. We found an acute effect of nicotine withdrawal ameliorating dyskinetic movements. Cross-sectional studies have suggested that the risk of tardive dyskinesia (TD) may be greater among smokers versus nonsmokers with schizophrenia (Yassa et al. 1987). Wirshing et al. (1989) found that acute inhalation of nicotine increased the severity of TD symptoms. In addition, a recent report presented epidemiological data suggesting that cigarette smoking is a risk factor for dyskinesias, independent of medication exposure (Nilsson et al. 1997). It is also interesting that the increase in dyskinesias occurred with nicotine replacement, although baseline nicotine levels were not maintained. Coupled with the observation that chronic nicotine exposure results in desensitization of the nicotinic receptor (McGhee and Role 1995), we might infer that the pharmacokinetics of nicotine delivery, as well as the pharmacodynamics of the nicotinic receptor, are likely to be important.

It may be possible to reconcile the differential effects of nicotine on dyskinesias versus psychiatric symptoms. Acutely, nicotine increases striatal and cortical dopamine release (Nisell et al. 1996). Nicotine abstinence would conceivably reverse this effect. It is possible that acute neurotransmitter/receptor effects of nicotine abstinence occur in both dorsal striatum (implicated in movement disorder pathophysiology) and cortical and subcortical dopamine systems (implicated in the positive and negative symptoms in schizophrenia), but that the circuitry mediating positive and negative symptoms is more complex (e.g., relatively polysynaptic) and slower to manifest these effects as compared to motor effects, which may involve less complex circuitry. For example, it is known that nicotine transiently normalized auditory gating in schizophrenics and their first-degree relatives (Freedman et al. 1997). It is also possible that nicotine withdrawal might exacerbate these deficits in auditory gating. If such deficits ultimately ramify to psychotic symptoms (e.g., auditory 
hallucinations), the time course of such a phenomenon might conceivably be more than a few days.

In summary, our findings do not support a straightforward symptom self-medication model to explain the high prevalence of smoking and low rate of cessation among individuals with schizophrenia. Interestingly, nicotine may modulate striatal dopamine function manifested as dyskinesias, but these effects require further study to understand the influence of nicotine dose and route of delivery on CNS motor systems. Continued study of smoking behavior in schizophrenia holds the promise of at least two important opportunities: to understand more about this illness, and to suggest ways to treat a deleterious addictive behavior in a vulnerable population.

\section{ACKNOWLEDGMENTS}

This work was supported by a Research Advisory Group (RAG) Grant from the Department of Veterans Affairs to GWD and by GCRC Grant MO1-RR00042. Versions of this work were presented at the 150th annual meeting of the American Psychiatric Association, San Diego, California, May 1997, and the 3rd annual scientific conference of the Society for Research on Nicotine and Tobacco, Nashville, Tennessee, June, 1997. The authors acknowledge the assistance of Gina Baslock and Dr. Victoria Florante.

\section{REFERENCES}

Addington J, el-Guebaly N, Addington D, Hodgins D (1997): Readiness to stop smoking in schizophrenia. Can J Psychiat 42:49-52

Adler LE, Hoffer LD, Wiser A, Freedman R (1993): Normalization of auditory physiology by cigarette smoking in schizophrenic patients. Am J Psychiat 150:1856-1861

Andreasen NC (1982): Negative symptoms in schizophrenia. Arch Gen Psychiat 39:784-788

Benowitz NL (1988): Pharmacologic aspects of cigarette smoking and nicotine addiction. NEJM 319:1318-1330

Covey L, Hughes DC, Glassman AH, Blazer DG, George LK (1994): Ever-smoking, quitting, and psychiatric disorders: Evidence from the Durham, North Carolina, Epidemiologic Catchment Area. Tobacco Control 3:222-227

Dalack GW, Meador-Woodruff JH (1996): Smoking, smoking withdrawal, and schizophrenia: Case reports and a review of the literature. Schizophr Res 22:133-141

Davis JM (1976): Comparative doses and costs of antipsychotic medication. Arch Gen Psychiat 33:858-861

de Leon J, Dadvand M, Canuso C, White AO, Stanilla JK, Simpson GK (1995): Schizophrenia and smoking: An epidemiological survey in a state hospital. Am J Psychiat 152:453-455

Decina P, Caracci G, Sandik R, Berman W, Mukherjee S, Scapicchio P (1990): Cigarette smoking and neurolepticinduced parkinsonism. Biol Psychiat 28:502-508
Ereshefsky L, Jann MW, Saklad SR, Davis CM, Richards AL, Burch NR (1985): Effects of smoking on fluphenazine in psychiatric inpatients. Biol Psychiat 20:329-332

Freedman R, Coon H, Myles-Worsley M, Orr-Urtreger A, Olincy A, Davis A, Polymeropoulos M, Holik J, Hopkins J, Hoff M, Rosenthal J, Waldo MC, Reimherr F, Wender P, Yaw J, Young DA, Breese CR, Adams C, Patterson D, Adler LE, Kruglyak L, Leonard S, Byerley W (1997): Linkage of a neurophysiological deficit in schizophrenia to a chromosome 15 locus. Proc Natl Acad Sci USA 94:587-592

Glassman AH (1993): Cigarette smoking: Implications for psychiatric illness. Am J Psychiat 150:546-553

Goff DC, Henderson DC, Amico E (1992): Cigarette smoking in schizophrenia: Relationship to psychopathology and medication side effects. Am J Psychiat 149:1189-1194

Hamilton M (1960): A rating scale for depression. J Neurol Neurosurg Psychiat 23:56-62

Hariharan M, VanNoord T, Greden JF (1988): A high-performance liquid chromatographic method for routine simultaneous determination of nicotine and cotinine in plasma. Clin Chem 34:724-729

Hatsukami DK, Hughes JR, Pickens RW, Svikis D (1984): Tobacco withdrawal symptoms: An experimental analysis. Psychopharmacology 84:231-236

Heatherton TF, Kozlowski LT, Frecker RC, Fagerstrom K-O (1991): The Fagerstrom Test for Nicotine Dependence: a revision of the Fagerstrom Tolerance Questionnaire. Brit J Addict 86:1119-1127

Hedlund JL, Vieweg BW (1980): The Brief Psychiatric Rating Scale (BPRS): A comprehensive review. J Operational Psychiat 11:48-64

Hughes JR, Hatsukami D (1986): Signs and symptoms of tobacco withdrawal. Arch Gen Psychiat 43:289-294

Hughes JR, Hatsukami DK, Mitchell JE, Dahlgren LA (1986): Prevalence of smoking among psychiatric outpatients. Am J Psychiat 413:993-997

Hughes JR, Higgins ST, Bickel WK (1994): Common errors in the pharmacologic treatment of drug dependence and withdrawal. Comp Ther 20:89-94

Jann MW, Saklad SR, Ereshefsky L, Richards AL, Harrington CA, Davis CM (1986): Effects of smoking on haloperidol and reduced haloperidol plasma concentrations and haloperidol clearance. Psychopharmacology 90:468-470

Krystal JH, Karper LP, Seibyl JP, Freeman GK, Delaney R, Bremner JD, Heninger GR, Bowers MB, Charney DS (1994): Subanesthetic effects of the noncompetitive NMDA antagonist, ketamine, in humans. Arch Gen Psychiat 51:199-214

McGhee DS, Role LW (1995): Physiological diversity of nicotinic acetylcholine receptors expressed by vertebrate neurons. Ann Rev Physiol 57:521-546

Menza MA, Grossman N, Van Horn M, Cody R, Forman N (1991): Smoking and movement disorders in psychiatric patients. Biol Psychiat 30:109-115

Munetz MR, Benjamin S (1988): How to examine patients using the Abnormal Involuntary Movement Scale. Hosp Comm Psychiat 39:1172-1177 
Nilsson A, Waller L, Rosengren A, Adlerberth A, Wilhelmsen L (1997): Cigarette smoking is associated with abnormal involuntary movements in the general male population-A study of men born in 1933. Biol Psychiat 41:717-723

Nisell M, Nomikos GG, Hertal P, Panagis G, Svensson TH (1996): Condition-independent sensitization of locomotor stimulation and mesocortical dopamine release following chronic nicotine treatment in the rat. Synapse 22:369-381

Nisell M, Nomikos GG, Svensson TH (1995): Nicotine dependence, midbrain dopamine systems, and psychiatric disorders. Pharmacol \& Toxicol 76:157-162

Overall JE, Gorham DR (1962): The Brief Psychiatric Rating Scale. Psychol Rep 10:799-812

Pickworth WB, Bunker EB, Henningfield JE (1994): Transdermal nicotine: Reduction of smoking with minimal abuse liability. Psychopharmacology 115:9-14

Psychopharmacology Research Branch, National Institute of Mental Health (1976): Abnormal Involuntary Movement Scale (AIMS). In Guy W (ed), ECDEU Assessment Manual for Psychopharmacology, revised. Rockville, MD, National Institute of Mental Health, US Dept Health, Education, and Welfare publication (ADM)76338, pp 534-537
Sanberg PR, Silver AA, Shytle RD, Philipp MK, Cahill DW, Fogelson HM, McConvile BJ (1997): Nicotine for the treatment of Tourette's syndrome. Pharmacol \& Therapeu 74:21-25

Sandyk R, Kay SR (1991): Tobacco addiction as a marker of age at onset of schizophrenia. Intern Neurosci 57:259262

Simpson GM, Angus JWS (1970): A rating scale for extrapyramidal side effects. Acta Psychiat Scand 212:11-19

Spitzer RL, Williams JBW, Gibbon M, First MB (1989): Structured Clinical Interview for DSM-III-R- Patient Version (SCID-P, 9/1/89 Version). New York, NY: Biometrics Research Department, New York State Psychiatric Institute

Wirshing WC, Engle J, Levin E, et al (1989): The acute effects of smoking on tardive dyskinesia. In 1989, New Research Program and Abstracts. San Francisco, CA: American Psychiatric Association

Yassa R, Lal S, Korpassy A, Ally J (1987): Nicotine exposure and tardive dyskinesia. Biol Psychiat 22:67-72

Ziedonis DM, George TP (1997): Schizophrenia and nicotine use: Report of a pilot smoking cessation program and review of neurobiological and clinical issues. Schizophr Bull 23:247-254 